\title{
Interdepartmental Spread of Innovations: A Multicentre Study of the Enhanced Recovery After Surgery Programme
}

\author{
Jeanny J. A. de Groot ${ }^{1,2}$ - José M. C. Maessen ${ }^{1,3}$ - Cornelis H. C. Dejong ${ }^{4,5,6}$ • \\ Bjorn Winkens $^{7}$ - Roy F. P. M. Kruitwagen ${ }^{2,6} \cdot$ Brigitte F. M. Slangen $^{2,6}$. \\ Trudy van der Weijden ${ }^{1} \cdot$ on behalf of all the members of the study group
}

Published online: 31 January 2018

(C) The Author(s) 2018. This article is an open access publication

\begin{abstract}
Background Spread of evidence-based innovations beyond pioneering settings is essential to improve quality of care. This study aimed to evaluate the influence of a national project to implement 'Enhanced Recovery After Surgery' (ERAS) among colorectal teams on the spread of this innovation to gynaecological procedures.

Methods A retrospective observational multicentre study was performed of a consecutive sample of patients who underwent major elective gynaecological surgery in 2012-2013. Ten Dutch hospitals (294 patients) had participated in a colorectal breakthrough project implementing ERAS on a nationwide basis and were assigned to the intervention group. Thirteen hospitals (390 patients) that had not participated in this project acted as controls. Outcome measures were time to functional recovery and total length of postoperative hospital stay. Multilevel models adjusted for clustering and baseline demographics were used for analysis. The uptake of ten selected perioperative care elements was evaluated for each hospital.

Results The estimated mean difference (95\% confidence interval) between the intervention and control hospitals was $-0.3(-0.9$ to 0.3$)$ days in the time to recovery and $0.2(-0.8$ to 1.3$)$ days in the total length of hospital stay. The mean ( \pm standard deviation) absolute rate of implemented perioperative care elements per hospital was $28.9 \pm 14.9 \%$ in the control, versus $29.3 \pm 11.1 \%$ in the intervention group $(p=0.934)$.

Conclusion Initial implementation effects seem to be restricted to the participating teams and do not automatically spread to other surgical teams in the same hospital.
\end{abstract}

The paper is based on a previous communication of the same data set, presented as a poster presentation at the 4th ERAS World Congress on Enhanced Recovery After Surgery held on 27-30 April 2016, Lisbon, Portugal.

Jeanny J. A. de Groot

jeanny.de.groot@mumc.nl

1 Department of Family Medicine, CAPHRI, School for Public Health and Primary Care, Maastricht University, P.O. Box 616, 6200 MD Maastricht, The Netherlands

2 Department of Obstetrics and Gynaecology, Maastricht University Medical Centre, P.O. Box 5800,

6202 AZ Maastricht, The Netherlands

\section{Introduction}

Innovations in healthcare are essential to ensure progress and to achieve the highest quality of care [1]. Despite the potential impact on outcomes, change of practice is

3 Department of Quality and Safety, Maastricht University Medical Centre, P.O. Box 5800, 6202 AZ Maastricht, The Netherlands

4 Department of Surgery, Maastricht University Medical Centre, P.O. Box 5800, 6202 AZ Maastricht, The Netherlands

5 NUTRIM - School for Nutrition and Translational Research in Metabolism, Maastricht University Medical Centre, P.O. Box 5800, 6202 AZ Maastricht, The Netherlands 
generally slow and challenging [2]. Quality improvement (QI) programmes are widely used to achieve change by applying a systematic approach. A growing body of evidence on their effectiveness has become available in recent years [3]. QI programmes, such as the collaborative breakthrough method [4, 5], provide support to spread innovations between stakeholders during the improvement process [6]. Spread of health innovations beyond this pioneering setting is necessary to continue improving quality of care. Studies have recognised that spread is also important to sustain innovation effects after an implementation project has ended [7]. Nevertheless, improvements often remain adopted by a restricted group of innovators [8].

Greenhalgh et al. [9] described a continuum between unplanned (diffusion) and planned (dissemination) mechanisms to achieve spread. Following this broad concept, we defined spread as the process through which effective innovations are adopted from one setting to another. Spread of clinical knowledge between departments can be hampered by intra-organisational barriers, such as group membership [10, 11]. A multiple case-study demonstrated that hospitals are able to spread both processes and content to other medical-surgical units using deliberate and active approaches to change (dissemination) [12]. Limited knowledge exists about the full continuum of local spread mechanisms when dissemination is not promoted and supported at a higher, national level.

We hypothesised that the experiences and tools gained after running a QI project among colorectal surgical teams would stimulate spread of a universal perioperative innovation to another closely related intra-organisational department, such as the gynaecological department. To examine this influence, we compared perioperative care and outcomes of gynaecological procedures in hospitals in which the colorectal surgical teams had taken part in a QI project [13], with the procedures performed by gynaecologists in hospitals in which colorectal surgical teams had not participated in the QI project.

6 GROW - School for Oncology and Developmental Biology, Maastricht University Medical Centre, P.O. Box 5800, 6202 AZ Maastricht, The Netherlands

7 Department of Methodology and Statistics, CAPHRI, School for Public Health and Primary Care, Maastricht University, P.O. Box 616, 6200 MD Maastricht, The Netherlands
Materials and methods

\section{Design}

A 1-year breakthrough project was used to implement 'Enhanced Recovery After Surgery' (ERAS) across 33 colorectal surgery departments in the Netherlands between 2006 and 2009 [13]. A significant change in practice was achieved, resulting in reduced length of hospital stay [13]. The spread from colorectal to gynaecological surgery was selected for analysis in the current retrospective multicentre study. The gynaecology and colorectal surgical teams were closely related and specialists cooperated regularly during surgical procedures, but departments operated independently. This observational study was exempt from ethics review (Medical Ethical Committee of the Maastricht University, METC13-5-031 and METC14-5-083).

\section{Inclusion criteria}

Dutch hospitals authorised to carry out major gynaecological cancer surgery were invited to participate. In the Netherlands, hospitals are required to meet a minimum number of 20 major ovarian cancer surgical procedures per year for authorisation. Both university and non-university teaching hospitals have the capacity to fulfil this criterion. The hospitals that had participated in the colorectal breakthrough project were included in the intervention group. Authorised hospitals that had not participated in an international or national project implementing ERAS for colorectal surgery acted as controls. One hospital participated in an international collaboration to develop and implement ERAS [14] and took the lead as an expert centre in the national implementation of ERAS in colorectal surgery [13]. As such, this hospital did not fulfil the criteria for the intervention or the control group and was excluded. The spread of ERAS towards gynaecology within this pioneering centre and the effects after active implementation have been described elsewhere [15]. The hospitals that regularly refer patients to an extended care facility for inpatient rehabilitation after surgery were excluded to prevent biased outcome measures. Hospitals with a combined ward for gynaecological and colorectal surgery could participate, but were registered as such.

A consecutive sample of patients who underwent elective open surgery were audited. Eligible patients were aged at least 18 years and had a suspected or proven diagnosis of ovarian, uterine, or cervical cancer. Surgical procedures included open exploratory, staging, and cytoreductive procedures in 2012 and 2013. 
Table 1 ERAS elements included in retrospective analysis

\begin{tabular}{ll}
\hline Preoperative phase & Omission of mechanical bowel preparation \\
Operative phase & Use of carbohydrate load \\
Use of local analgesia & Avoidance of drains \\
Postoperative phase & Early ambulation on the day of surgery (sitting on edge of bed) \\
Scheduled postoperative administration of laxatives & Oral feeding on POD 1 \\
Early ambulation on POD 1 (sitting on chair three times a day) & Discontinuation of intravenous fluids on POD 2 \\
Discontinuation of urinary catheter on POD 2
\end{tabular}

ERAS Enhanced Recovery After Surgery, $P O D$ postoperative day

\section{Description of the innovation}

The uptake of the multifaceted ERAS guideline was evaluated. Based on the best available evidence, ERAS consists of several perioperative recommendations to reduce time of recovery after surgery [16]. Although originally developed for colorectal surgery, the programme is not exclusive to this specialty [17]. To date, more surgical specialties, such as gastric [18] and pancreatic surgery [19], urology [20], and gynaecology [21, 22], have adopted ERAS and have tailored guidelines to specific procedures or to local policies [23]. Individual elements of ERAS, such as the avoidance of drains, have been shown to be effective in gynaecological surgery for several years [24-28].

\section{Outcome measures}

Primary outcomes were the time to functional recovery after surgery (FR) and the total length of postoperative hospital stay (TLOS) in days. Recovery was reached when patients tolerated oral food and oral analgesia and regained their mobility. TLOS included the number of nights a patient stayed in the hospital after surgery plus, if appropriate, the length of readmission. The secondary outcome included the degree of local implementation of single ERAS elements; for each hospital, this was dichotomised using a target of $70 \%$ adherence as a minimum degree of implementation [29, 30]. A combined score per element was calculated. Mortality and readmission rates were registered.

\section{Data collection}

Retrospective review of medical records was performed up to 30 days postoperatively. In considering the Dutch volume norm, a maximum of 30 patients per hospital were audited. Hospital types were categorised as university and non-university teaching hospitals. There is one specialised cancer centre, which was categorised as a university hospital. The type of gynaecological cancer, histological subtype, and type of incision were specified. To obtain accurate information, data of ten ERAS elements were registered (Table 1). These elements covered the three perioperative phases of care, and their combination represents an ERAS management.

\section{Data analysis}

A descriptive analysis of baseline characteristics was performed. For categorical variables, differences were analysed using the $\chi^{2}$ test or Fisher's exact test. The independentsamples $t$ test was used for numerical variables. To account for clustering of patients within hospitals, linear multilevel methods were used to evaluate differences in time to FR and TLOS. The models incorporated hospital identification as a random effect, whereas study group, hospital type, presence of a combined ward, age $(<60$ versus $\geq 60)$, American Society of Anaesthesiologists classification, type of cancer, histological subtype, and type of incision were included as fixed effects. Missing outcome values were not imputed, since likelihood-based methods were used. The estimated marginal means (EMM) and mean differences (MD), corresponding $95 \%$ confidence intervals $(\mathrm{CI})$, and intra-class correlation coefficients (ICC) were presented. Unadjusted differences in the median time to FR and TLOS were analysed at hospital level using the Mann-Whitney U test because of the relatively small number of hospitals (13 versus 10 ). A reduction in time to FR and TLOS of more than 1 day was assumed to be clinically relevant. A value of $p \leq 0.005$ was considered statistically significant. Analyses were performed with IBM SPSS Statistics for Windows, version 21.0 (Armonk, NY, USA).

\section{Results}

A total of 684 medical records from 23 hospitals were audited (Fig. 1). Ten hospitals had followed the breakthrough strategy for colorectal surgery and were allocated 
Fig. 1 Study flow chart

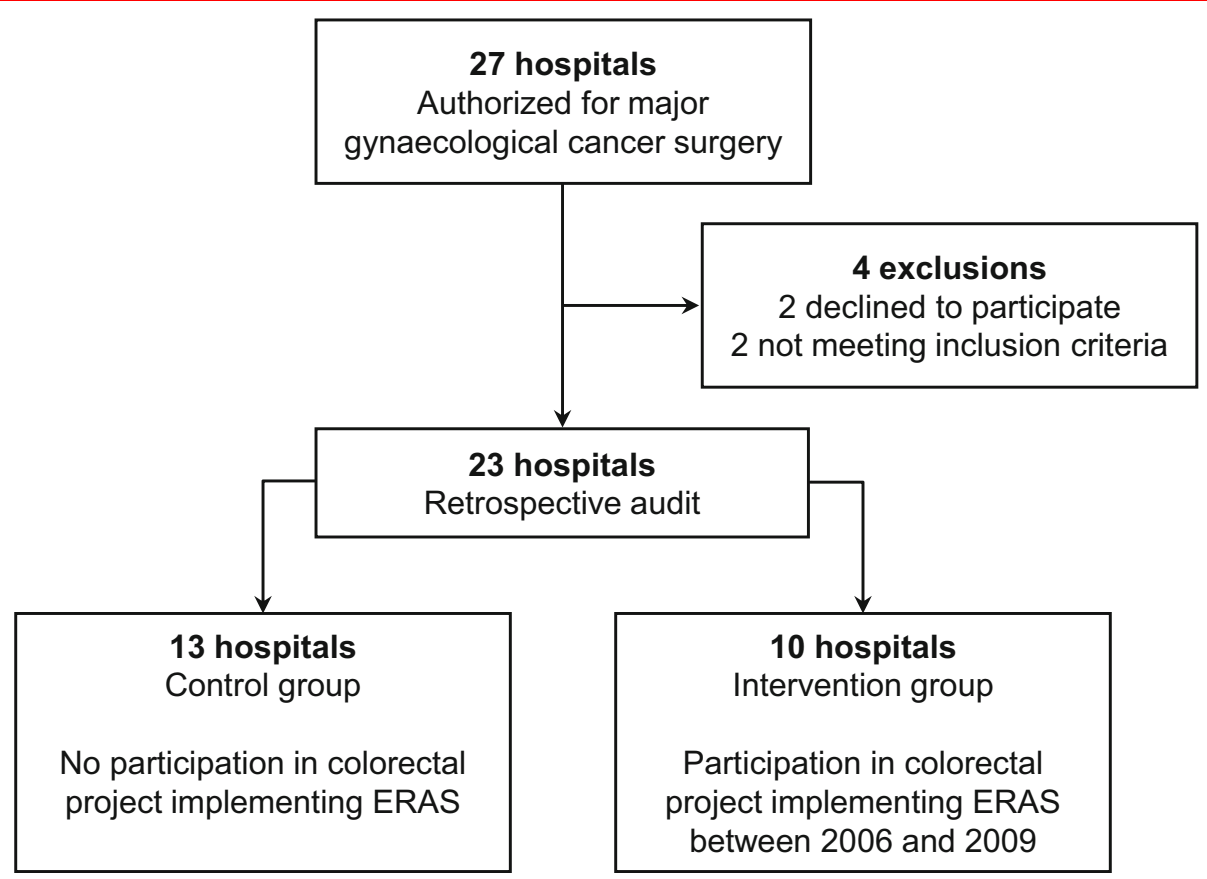

to the intervention arm. The other hospitals were regarded as controls $(n=13)$. There were no statistically significant between-group differences in demographic data regarding the hospitals (Table 2). Some differences in the indication for surgery were noted between patient groups.

FR was registered for $97.2 \%$ of the patients in the control and for $98.6 \%$ in the intervention group. Data on the time to FR were missing in five patients in the control and in one patient in the intervention group. In addition, two patients in the control group were transferred to another hospital before recovery was reached. These patients were lost to follow up. Mortality rate was comparable between the control $(n=4,1.0 \%)$ and intervention $(n=3,1.0 \%)$ groups $(p>0.999)$. The hospitals' median time to FR was 4.0 days (between-hospital range 3.0-6.0) in the control group, and 4.0 days (between-hospital range $3.0-5.0)$ in the intervention group $(p=0.218)$. Following adjustment for baseline variables, multilevel modelling showed an MD of -0.3 days (95\% CI -0.9 to 0.3 ) in FR between patients treated in intervention hospitals compared to treatment in a control hospital. The EMM was 4.2 days (95\% CI 3.4-5.0) in the control group compared to 3.9 days $(95 \%$ CI 3.1-4.8) in the intervention group $(p=0.319$ ) (Fig. 2). The ICC for FR was 0.03 .

The median TLOS was 6.0 days in the control hospitals (between-hospital range 4.0-7.0) and 5.5 days for hospitals in the intervention group (between-hospital range 3.5-10.5) $(p=0.777)$. The observed effect remained statistically nonsignificant after adjusting for covariates (MD 0.2 days; $95 \% \mathrm{CI}-0.8$ to 1.3$)$. The EMM was 5.8 days $(95 \% \mathrm{CI}$
4.4-7.2) in the control group compared to 6.1 days $(95 \%$ CI 4.6-7.5) in the intervention group (Fig. 2). The ICC was 0.06 . In total, 12 patients $(3.1 \%)$ from the control and 14 patients $(4.8 \%)$ in the intervention group had to be readmitted $(p=0.254)$.

The degree of implementation within hospitals was based on the adherence to ten selected ERAS elements. Omission of mechanical bowel preparation, the use of local analgesia, and the avoidance of abdominal drains were the most common elements adhered to in both study groups (Fig. 3). Adoption of postoperative ERAS elements was low. No statistically significant differences were found between groups. In the control group, three hospitals (23\%) implemented at least half the elements in daily practice compared to one hospital $(10 \%)$ in the intervention group $(p=0.604)$. None of these were hospitals with a combined ward for colorectal and gynaecological surgery patients. The mean absolute rate of implemented elements per hospital was $28.9 \%$ (SD $14.9 \%$ ) in the control and $29.3 \%$ (SD 11.1\%) in the intervention group ( $p=0.934)$.

\section{Discussion}

Despite the acquired knowledge and positive experiences gained from the implementation of ERAS in colorectal surgery, this study showed that almost no spread took place in the closely related department of gynaecology in the 5 years following implementation. Multilevel regression analysis, taking clustering of hospitals and baseline 
Table 2 Baseline demographics of hospitals and patients according to observational group

\begin{tabular}{|c|c|c|c|}
\hline & Control & Intervention & $p$ value $^{\mathrm{b}}$ \\
\hline \multicolumn{4}{|l|}{ Hospital demographics } \\
\hline Number of hospitals & 13 & 10 & \\
\hline Number of beds per hospital ${ }^{\mathrm{a}}$ & $642.3 \pm 304.5$ & $812.1 \pm 189.8$ & $0.138^{\mathrm{c}}$ \\
\hline Type of hospital & & & $0.660^{\mathrm{d}}$ \\
\hline University medical centre & $4(30.8)$ & $2(20.0)$ & \\
\hline Non-university teaching hospital & $9(69.2)$ & $8(80.0)$ & \\
\hline \multicolumn{4}{|l|}{ Hospital ward } \\
\hline Combined with colorectal surgery & $1(7.7)$ & $2(20.0)$ & $0.560^{\mathrm{d}}$ \\
\hline \multicolumn{4}{|l|}{ Patient demographics } \\
\hline Number of patients & 390 & 294 & \\
\hline Age, years ${ }^{\mathrm{a}}$ & $61.4 \pm 12.0$ & $62.6 \pm 12.4$ & $0.212^{\mathrm{c}}$ \\
\hline Age, years & & & 0.016 \\
\hline$<60$ & $164(42.1)$ & $97(33.0)$ & \\
\hline$\geq 60$ & $226(57.9)$ & $197(67.0)$ & \\
\hline ASA classification & & & 0.092 \\
\hline Class I/II & $352(90.3)$ & $253(86.1)$ & \\
\hline Class III/IV & $38(9.7)$ & $41(13.9)$ & \\
\hline Gynaecological cancer type & & & 0.045 \\
\hline Ovarian & $289(74.1)$ & $231(78.6)$ & \\
\hline Uterine & $72(18.5)$ & $54(18.4)$ & \\
\hline Cervical & $29(7.4)$ & $9(3.1)$ & \\
\hline Histological subtype & & & 0.981 \\
\hline Benign & $46(11.8)$ & $36(12.2)$ & \\
\hline Borderline/hyperplasia & $30(7.7)$ & $22(7.5)$ & \\
\hline Malignant & $314(80.5)$ & $236(80.3)$ & \\
\hline Type of incision & & & 0.842 \\
\hline Midline & 377 (96.7) & $285(96.9)$ & \\
\hline Transverse & $13(3.3)$ & $9(3.1)$ & \\
\hline
\end{tabular}

Values in parentheses are percentages unless indicated otherwise

ASA American society of anaesthesiologists

${ }^{\mathrm{a}}$ Values are mean \pm standard deviation. ${ }^{\mathrm{b}} \chi^{2}$ test, except ${ }^{\mathrm{c}}$ independent samples $t$ test and ${ }^{\mathrm{d}}$ Fisher's exact test

demographics into account, demonstrated no differences in outcomes between gynaecological procedures performed in hospitals that had participated in the colorectal QI project, and the hospitals that did not. Even the hospitals with a mixed ward for colorectal and gynaecological surgery did not adopt the majority of ERAS elements.

None of the single ERAS elements was regularly adopted by all the hospitals, despite the clear evidence already available for many years [21, 22]. This finding confirms the outcomes of a single centre study in an earlyadopter hospital [15] and emphasises the challenge to spread and actively implement ERAS in actual practice [31]. Greenhalgh and colleagues [9] have described several characteristics of innovations that are required to facilitate spread. Although the multifaceted and behaviour changing aspects of ERAS may render implementation in actual practice difficult, the innovation is particularly suitable to

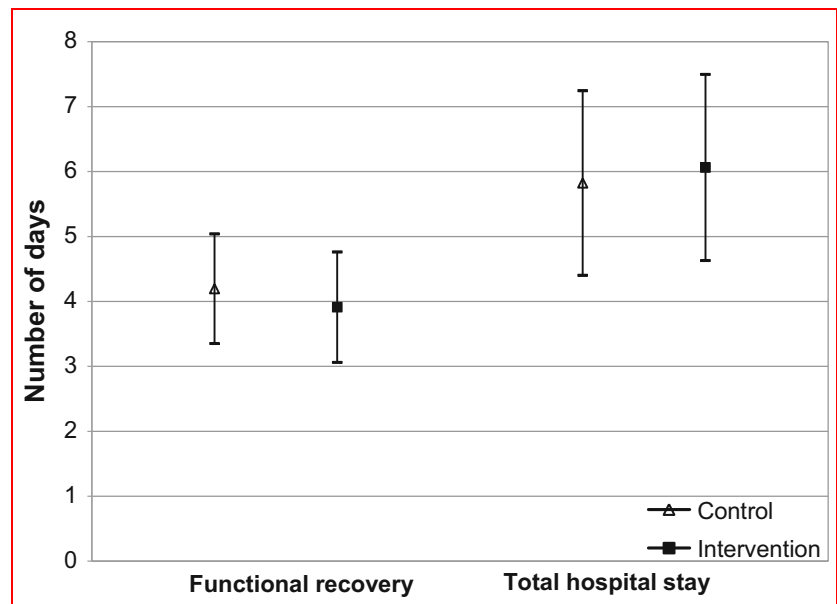

Fig. 2 Model estimated marginal means in days 
Fig. 3 Percentage of implemented $(>70 \%)$ enhanced recovery elements among hospitals

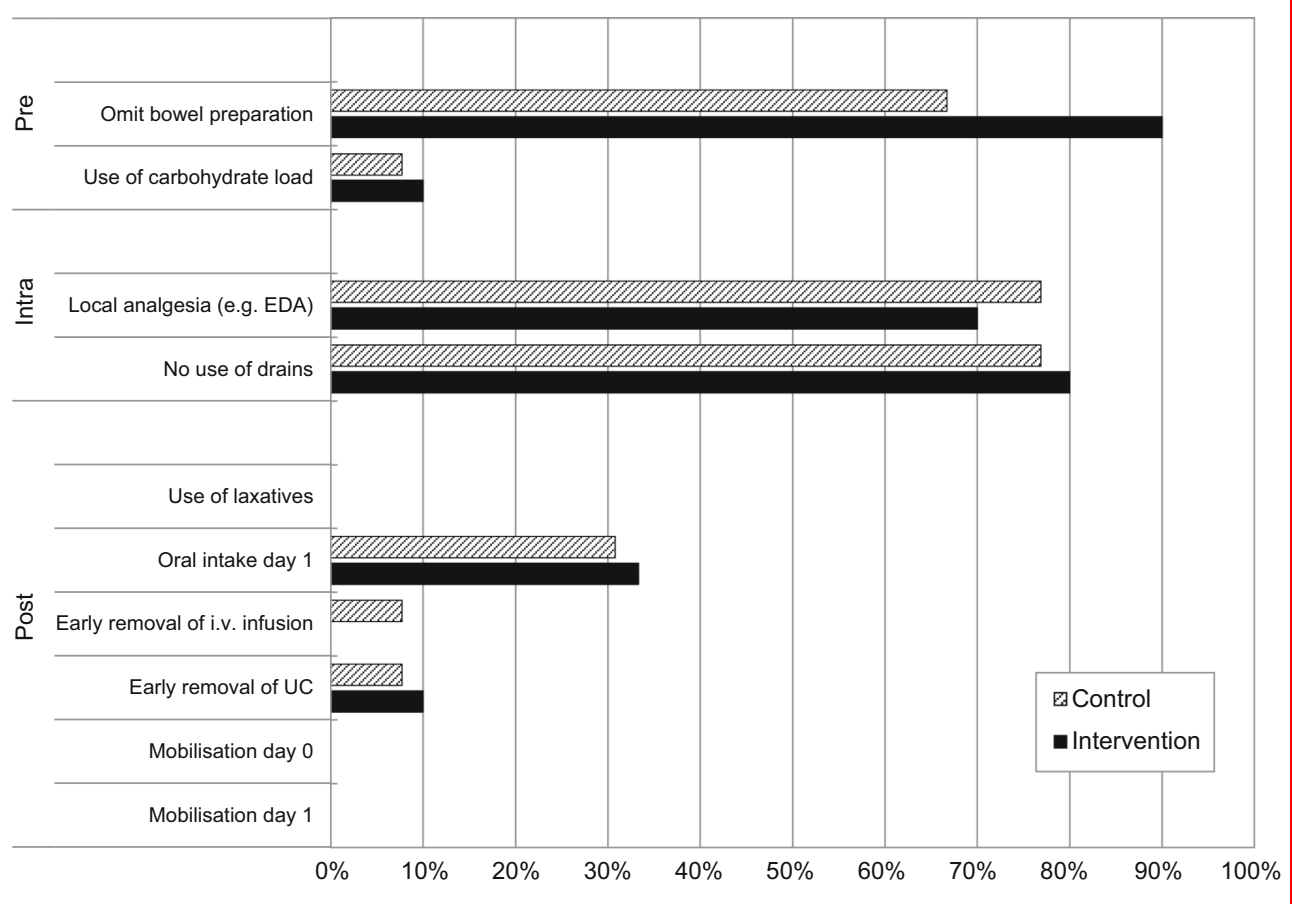

spread within organisations. ERAS principles are universal, and effectiveness has been demonstrated [32-34]. Despite the complex multifaceted structure, implementation can be achieved by using small, manageable steps. Benefits are almost directly visible for both the innovators and patients. Furthermore, the need for a multidisciplinary approach should facilitate intra-organisational spread.

This study included hospitals that participated in the original breakthrough strategy [13]. The breakthrough strategy is designed to spread innovations across multiple organisations over a short period of time [4]. Participants learned to apply practical skills to improve care, and all participating hospitals reduced length of hospital stay and/ or had high adherence rates [13]. Therefore, the expertise acquired should give them the capacity to guide local spread. In this study, we tried to analyse the full continuum of local spread without promotion and guidance at a higher, national level. None of the hospitals achieved a complete spread of ERAS, and just one hospital in the intervention group implemented at least half of the ERAS elements. Unfortunately, we lack consistent information about the sustainability of ERAS in colorectal surgery in those hospitals.

Labgaa et al. [35] recently noted that structured implementation of ERAS in one speciality (colorectal surgery) induced a transition of elements towards another speciality (liver surgery) within the same department in patients treated by the same team. Evidence is limited about the effect of conducting a systematic QI programme on the spread of innovations between departments. It has been shown frequently that changing behaviour within healthcare organisations is challenging and complex [2, 3, 36]. Although results need to be interpreted with caution, we believe this study emphasises those findings and shows that barriers are even higher than generally expected. Awareness about the barriers might be the first step in breaking through apparent boundaries and creating a culture of change. During recent years, the shift from a traditional function-oriented towards a process-oriented structure of hospitals has gained attention [37]. The functional grouping of departments in the Netherlands seems to hamper the multidisciplinary efforts to optimise patient care. Breaking through this group membership facilitates knowledge sharing between different groups of healthcare providers. Furthermore, the involvement of intra-organisational knowledge brokers may promote spread of innovations across health-related sectors [38]. A recent review described knowledge brokers as intermediaries to exchange knowledge across the varying stakeholders and settings, but demonstrated that the availability of evidence about their effectiveness within healthcare is limited so far [39]. In line with other studies, we found a gap in discharge [40, 41]. Unnecessary prolonged hospitalisation might be partially reduced by applying structured discharge planning, or by using an integrated discharge team [42].

The multicentre design strengthens conclusions and provides more generalisability of results. To our knowledge, the national comparison of interdepartmental spread between hospitals that had previously followed a QI programme, and those that had not, is unique. A limitation 
might be that the data abstractor was not blinded. We do not have any sign, however, that this has led to data manipulation, given the independent position. Otherwise, the fact that the data were extracted by one and the same person can be looked upon as a strength, as it guaranteed a standardised approach for the audit. Retrospective review of medical records has methodological limitations. The adoption of ERAS could have been underestimated, because not all elements could be collected retrospectively. It is possible that we had inadequate power to detect significant differences. However, given the $95 \%$ CIs, we would have been able to detect a clinically relevant effect of more than 1 day favouring the intervention group [43].

In conclusion, we found neither statistically significant nor clinically relevant differences in the time to FR, TLOS, or the degree of local implementation of ERAS, between the gynaecology departments of hospitals that previously followed a QI project for colorectal surgery and the hospitals that did not. Interdepartmental spread of universal evidence-based innovations within organisations seems to be restricted. Further research is required to provide potential solutions to promote knowledge sharing within hospital walls and to extend the initial positive effects of QI projects to different settings.

Acknowledgements This research project was financially supported by The Netherlands Organisation for Health Research and Development, ZonMw, Grant Number 80-83700-98-133021. The funders had no role in study design, data collection and analysis, decision to publish, or preparation of the manuscript.

The members of the group meet the criteria for authorship. Local leads of the study group: R.L.M. Bekkers (Radboud University Nijmegen Medical Centre, Nijmegen; Catharina Hospital, Eindhoven); E.A. Boss (Maxima Medical Centre, Veldhoven); E.B.L. van Dorst (University Medical Centre Utrecht, Utrecht); W.J. van Driel (Netherlands Cancer Institute, Amsterdam); G. Fons (Academic Medical Centre, Amsterdam); K.N. Gaarenstroom (Leiden University Medical Centre, Leiden); C.G. Gerestein (Meander Medical Centre, Amersfoort); M. van Haaften (Diakonessenhuis, Utrecht); A.M.L.D. van Haaften-de Jong (HagaZiekenhuis, The Hague); H.H. de Haan (Isala Klinieken, Zwolle); D. van Hamont (Amphia Hospital, Breda); R.H.M. Hermans (Catharina Hospital, Eindhoven); W. Hofhuis (Franciscus Hospital, Rotterdam); L.N. Hofman (Albert Schweitzer Hospital, Dordrecht); J.E. Martens (Maasstad Hospital, Rotterdam); H. Mertens (Zuyderland Hospital, Sittard-Geleen); B.M. Pijlman (Jeroen Bosch Hospital,'s Hertogenbosch); J.M.A. Pijnenborg (Elisabeth-TweeSteden Hospital, Tilburg; Radboud University Nijmegen Medical Centre, Nijmegen); N. Reesink-Peters (Medisch Spectrum Twente, Enschede); E.M. Roes (Erasmus Medical Centre, Rotterdam); J.H. Schagen van Leeuwen (St Antonius Hospital, Nieuwegein); M.P.L.M. Snijders (Canisius Wilhelmina Hospital, Nijmegen); P.M.L.H. Vencken (Bravis Hospital, Bergen op Zoom).

\section{Compliance with Ethical Standards}

Conflict of interest The authors declare that they have no conflict of interest.
Open Access This article is distributed under the terms of the Creative Commons Attribution 4.0 International License (http://crea tivecommons.org/licenses/by/4.0/), which permits unrestricted use, distribution, and reproduction in any medium, provided you give appropriate credit to the original author(s) and the source, provide a link to the Creative Commons license, and indicate if changes were made.

\section{References}

1. Riskin DJ, Longaker MT, Gertner M et al (2006) Innovation in surgery: a historical perspective. Ann Surg 244:686-693

2. Grimshaw JM, Eccles MP, Lavis JN et al (2012) Knowledge translation of research findings. Implement Sci 7:50

3. Grimshaw JM, Thomas RE, MacLennan G et al (2004) Effectiveness and efficiency of guideline dissemination and implementation strategies. Health Technol Assess 8:iii-iv:1-72

4. Institute for Healthcare Improvement (2003) The breakthrough series: IHI's collaborative model for achieving breakthrough improvement. IHI Innovation Series White Paper, Institute for Healthcare Improvement, Boston

5. Kilo CM (1998) A framework for collaborative improvement: lessons from the Institute for Healthcare Improvement's Breakthrough Series. Qual Manag Health Care 6:1-13

6. Ovretveit J, Klazinga N (2012) Learning from large-scale quality improvement through comparisons. Int J Qual Health Care $24: 463-469$

7. Slaghuis SS, Strating MM, Bal RA et al (2013) A measurement instrument for spread of quality improvement in healthcare. Int $\mathbf{J}$ Qual Health Care 25:125-131

8. Massoud MR, Nielsen GA, Nolan K et al (2006) A framework for spread: from local improvements to system-wide change. IHI Innovation Series white paper, Institute for Healthcare Improvement, Cambridge

9. Greenhalgh T, Robert G, Macfarlane F et al (2004) Diffusion of innovations in service organizations: systematic review and recommendations. Milbank Q 82:581-629

10. Tasselli S (2014) Social networks of professionals in health care organizations: a review. Med Care Res Rev 71:619-660

11. Currie G, Waring J, Finn R (2008) The limits of knowledge management for UK public services modernization: the case of patient safety and service quality. Public Adm 86:363-385

12. Pearson ML, Upenieks VV, Yee T et al (2008) Spreading nursing unit innovation in large hospital systems. J Nurs Adm 38:146-152

13. Gillissen F, Hoff C, Maessen JM et al (2013) Structured synchronous implementation of an enhanced recovery program in elective colonic surgery in 33 hospitals in The Netherlands. World J Surg 37:1082-1093. https://doi.org/10.1007/s00268-0131938-4

14. Maessen J, Dejong CH, Hausel J et al (2007) A protocol is not enough to implement an enhanced recovery programme for colorectal resection. Br J Surg 94:224-231

15. de Groot JJ, van Es LE, Maessen JM et al (2014) Diffusion of enhanced recovery principles in gynecologic oncology surgery: is active implementation still necessary? Gynecol Oncol 134:570-575

16. Gustafsson UO, Scott MJ, Schwenk W et al (2013) Guidelines for perioperative care in elective colonic surgery: enhanced recovery after surgery $\left(\right.$ ERAS $\left.^{\circledR}\right)$ society recommendations. World J Surg 37:259-284. https://doi.org/10.1007/s00268-012-1772-0

17. Ljungqvist O, Scott M, Fearon KC (2017) Enhanced recovery after surgery: a review. JAMA Surg 152:292-298 
18. Mortensen K, Nilsson M, Slim K et al (2014) Consensus guidelines for enhanced recovery after gastrectomy: enhanced recovery after surgery $\left(\mathrm{ERAS}^{\circledR}\right)$ society recommendations. Br J Surg 101:1209-1229

19. Lassen K, Coolsen MM, Slim K et al (2013) Guidelines for perioperative care for pancreaticoduodenectomy: enhanced recovery after surgery $\left(\right.$ ERAS $\left.^{\circledR}\right)$ society recommendations. World J Surg 37:240-258. https://doi.org/10.1007/s00268-012-1771-1

20. Cerantola Y, Valerio M, Persson B et al (2013) Guidelines for perioperative care after radical cystectomy for bladder cancer: enhanced recovery after surgery $\left(\right.$ ERAS $^{\circledR}$ ) society recommendations. Clin Nutr 32:879-887

21. Nelson G, Altman AD, Nick A et al (2016) Guidelines for preand intra-operative care in gynecologic/oncology surgery: enhanced recovery after surgery $\left(\right.$ ERAS $\left.^{\circledR}\right)$ society recommendations-part I. Gynecol Oncol 140:313-322

22. Nelson G, Altman AD, Nick A et al (2016) Guidelines for postoperative care in gynecologic/oncology surgery: enhanced recovery after surgery $\left(\mathrm{ERAS}^{\circledR}\right.$ ) society recommendations-part II. Gynecol Oncol 140:323-332

23. Paton F, Chambers D, Wilson P et al (2014) Effectiveness and implementation of enhanced recovery after surgery programmes: a rapid evidence synthesis. BMJ Open 4:e005015

24. Benedetti-Panici P, Maneschi F, Cutillo G et al (1997) A randomized study comparing retroperitoneal drainage with no drainage after lymphadenectomy in gynecologic malignancies. Gynecol Oncol 65:478-482

25. Charoenkwan K, Kietpeerakool C (2014) Retroperitoneal drainage versus no drainage after pelvic lymphadenectomy for the prevention of lymphocyst formation in patients with gynaecological malignancies. Cochrane Database Syst Rev 6:CD007387

26. Franchi M, Trimbos JB, Zanaboni F et al (2007) Randomised trial of drains versus no drains following radical hysterectomy and pelvic lymph node dissection: a European organisation for research and treatment of cancer-gynaecological cancer group (EORTC-GCG) study in 234 patients. Eur J Cancer 43:1265-1268

27. Jensen JK, Lucci JA 3rd, DiSaia PJ et al (1993) To drain or not to drain: a retrospective study of closed-suction drainage following radical hysterectomy with pelvic lymphadenectomy. Gynecol Oncol 51:46-49

28. Lopes AD, Hall JR, Monaghan JM (1995) Drainage following radical hysterectomy and pelvic lymphadenectomy: dogma or need? Obstet Gynecol 86:960-963

29. Ahmed J, Khan S, Gatt M et al (2010) Compliance with enhanced recovery programmes in elective colorectal surgery. Br J Surg 97:754-758
30. Gustafsson UO, Hausel J, Thorell A et al (2011) Adherence to the enhanced recovery after surgery protocol and outcomes after colorectal cancer surgery. Arch Surg 146:571-577

31. Kahokehr A, Sammour T, Zargar-Shoshtari K et al (2009) Implementation of ERAS and how to overcome the barriers. Int $\mathbf{J}$ Surg 7:16-19

32. de Groot JJ, Ament SM, Maessen JM et al (2016) Enhanced recovery pathways in abdominal gynecologic surgery: a systematic review and meta-analysis. Acta Obstet Gynecol Scand 95:382-395

33. Barber EL, Van Le L (2015) Enhanced recovery pathways in gynecology and gynecologic oncology. Obstet Gynecol Surv 70:780-792

34. Nelson G, Kalogera E, Dowdy SC (2014) Enhanced recovery pathways in gynecologic oncology. Gynecol Oncol 135:586-594

35. Labgaa I, Jarrar G, Joliat GR et al (2016) Implementation of enhanced recovery (ERAS) in colorectal surgery has a positive impact on non-ERAS liver surgery patients. World J Surg 40:1082-1091. https://doi.org/10.1007/s00268-015-3363-3

36. Johnson MJ, May CR (2015) Promoting professional behaviour change in healthcare: what interventions work, and why? A theory-led overview of systematic reviews. BMJ Open 5:e08592

37. Vos L, Chalmers SE, Duckers ML et al (2011) Towards an organisation-wide process-oriented organisation of care: a literature review. Implement Sci 6:8

38. Waring J, Currie G, Crompton A et al (2013) An exploratory study of knowledge brokering in hospital settings: facilitating knowledge sharing and learning for patient safety? Soc Sci Med 98:79-86

39. Bornbaum CC, Kornas K, Peirson L et al (2015) Exploring the function and effectiveness of knowledge brokers as facilitators of knowledge translation in health-related settings: a systematic review and thematic analysis. Implement Sci 10:162

40. Maessen JM, Dejong CH, Kessels AG et al (2008) Length of stay, an inappropriate readout of the success of enhanced recovery programs. World J Surg 32:971-975. https://doi.org/10.1007/ s00268-007-9404-9

41. Fiore JF Jr, Faragher IG, Bialocerkowski A et al (2013) Time to readiness for discharge is a valid and reliable measure of shortterm recovery after colorectal surgery. World J Surg 37:2927-2934. https://doi.org/10.1007/s00268-013-2208-1

42. Gonçalves-Bradley DC, Lannin NA, Clemson LM et al (2016) Discharge planning from hospital. Cochrane Database Syst Rev 1:CD000313

43. Goodman SN, Berlin JA (1994) The use of predicted confidence intervals when planning experiments and the misuse of power when interpreting results. Ann Intern Med 121:200-206 